\title{
NFC based provisioning of instructional videos to assist with instrumental activities of daily living
}

\author{
Joseph Rafferty, Chris Nugent, Liming Chen, Jun Qi, Rachael Dutton, Anna Zirk, Lars Thomas Boye, \\ Michael Kohn, Ritta Hellman
}

\begin{abstract}
Existing assistive living and prompting based solutions have adopted a relatively complex approach to supporting individuals. These solutions have involved sensor based monitoring, activity recognition and assistance provisioning. Traditionally they have suffered from a number of issues rooted in scalability and performance levels associated with the activity recognition process. This paper introduces a simplistic approach to assistive living within a user's residence through the use of NFC tags and smart devices. The core concept of this approach is presented and is subsequently placed within the context of related work. A description of the architecture is provided and results following technical evaluation of the first system prototype are discussed.
\end{abstract}

\section{INTRODUCTION}

The worldwide population is transitioning to never before recorded, uneven, composition resulting in global aging [1]. This global aging is expected to produce a situation where by 2050 over $20 \%$ of the population will be aged 65 or over [2]. This change in population is expected to produce an increase in aging related illnesses which, in turn, are expected to place additional burdens on healthcare provision and its associated support infrastructure [2]. Additionally, this aging within the population will be accompanied with a reduction of the ratio of working aged people (15-64) to those over 65 , thus reducing the amount of informal support available [1].

The portion of the population suffering from these aging related illnesses will therefore require some form of support to live independently and remain within their own homes for as long as possible. Ambient Assistive Living (AAL) is a technological based approach which aims to support

Research supported by the EU AAL program.

J. Rafferty is with the University of Ulster, Jordanstown, Northern Ireland, BT37 0QB, UK (phone: 028-90368840; e-mail: raffertyj@email.ulster.ac.uk).

C. Nugent and J. Qi are with the University of Ulster, Jordanstown, Northern Ireland, BT37 0QB, UK (e-mail: cd.nugent@ulster.ac.uk, j.qi@ulster.ac.uk).

L. Chen is with De Montfort University, Leicester, LE1 9BH, UK (email: liming.chen@dmu.ac.uk).

R. Dutton is with Accord Group, West Midlands, B70 6QG, UK (email: rachael.dutton@accordgroup.org.uk).

A. Zirk is with Berliner Institut für Sozial-forschung, D-10707 Berlin, Germany (email: a.zirk@bis-berlin.de).

L. T. Boye is with Tellu AS, NO-1383 Asker, Norway (email: lars.thomas.boye@tellu.no).

M. Kohn is with Stickyworld PLC, London, EC2A 4BX, UK (email: michael@stickyworld.com)

R. Hellman is with Karde AS, PO Box 69 Tåsen, 0801 Oslo, Norway (email: riitta.hellman@karde.no) individuals with aged related impairments [3], [4], [5]. AAL has been widely viewed as a promising approach to address the problems associated with aging and subsequently increase the independence of individuals [5], [6]. One of the core elements within the domain of AAL focuses on solutions with the intention of assisting individuals with successfully completing both Activities of Daily Living (ADLs) [7] and Instrumental Activities of Daily Living (IADLs) [8].

Although a number of solutions exist which provide support for ADLs/IADLs, these solutions each come with advantages and disadvantages. The Personal IADL Assistant (PIA) project aims to address some of these weaknesses by producing a cloud-based, social-network augmented approach. This approach removes the complexity of current approaches and provides a scalable method of flexibly providing relatable video-based instruction.

The PIA approach provides assistance with IADLs by providing video based instruction following interaction of smart devices (i.e. a phone or tablet) with Near Field Communication (NFC) tags affixed to household objects.

The remainder of this paper is organized as follows; an overview of related work is provided in Section II. The approach used in the PIA project is explained in Section III. Section IV details the results from the initial evaluation of the system and conclusions and future work are presented in Section V.

\section{RELATED WORK}

A number of assistive systems currently exist to help individuals complete activities within a home environment. A large portion of these systems have been embedded within the Smart Homes (SH) paradigm with a number of approaches specifically offering prompting based support.

\section{A. Smart homes}

SHs are residential environments which have been augmented with a variety of technologies in order to provide their assistive function. Generally, SHs incorporate three layers of technology to provide their function [4], [5], [6], [9], [10]. These layers include; a sensor deployment in order to monitor inhabitants, a processing layer to support some form of activity recognition or prediction and actuators to provide assistance. Actuators can be used to assist inhabitants with completing activities that they are having difficulty with. They can provide support in the form of automation within the environment itself or they can support interfaces which allow prompting in order to guide an inhabitant. The sensor components of SHs may employ a 
large number of sensing elements ranging from contact switches affixed to items to passive infrared and ultrasonic based tracking mechanisms to computer vision based solutions.

Generally, signals from these sensors are processed by some form of activity recognition or prediction process to determine the unfolding of activities being undertaken. Activities detected are subsequently monitored to determine if an inhabitant is having difficulty with their completion. If difficulty is determined an assistive prompt would be issued. After prompts are issued further observation and recognition processes may occur to ensure the activity has been successfully completed, if not additional guidance may be supplied [5], [6], [9], [10], [11], [12].

These assistive systems have shown promise; however, they have suffered from a number of barriers to their widespread adoption. Most notably, by requiring widespread sensor deployments and an associated processing layer to interpret unfolding activities. In order to deploy these systems in a preexisting residence, a potentially large sensor suite would be required. This retrofitting represents an inconvenience from the physical environment perspective in addition to incurring a financial cost making this process unfeasible for wide scale deployments [3], [4], [5], [6], [9].

Additionally, there are some limitations with current activity recognition processes including, lack of capability to flexibly model activities, difficulties handling interleaved and concurrent activities and privacy concerns [4], [5], [6], [9].

\section{B. Prompting systems}

Prompting systems exist to provide guidance and reminders for their users given certain criteria. The prompts provided may be video, audio or text based. Of these types of prompt, video based instruction, particularly those with an audio component, provide a promising avenue to offer detailed and relatable guidance for users [5], [9], [10], [13], [14]. In particular, videos recorded by a trusted individual that a user may relate to can provide a sense of familiarity. This sense of familiarity is essential and provides important emotional contact with those who are providing care and also those who are receiving it [15].

One such system, $\mathrm{COACH}$ [10], was designed to support individuals suffering from moderate level dementia by assisting them with the complex hand washing ADL. This system uses a camera to monitor a user's hand washing activity. Images from this camera can then be translated into hand and towel positions by a tracking system. These positions are then passed to the activity recognition process with the aim of producing the current belief state. This belief state is associated with a policy that then determines how best to assist the user by prompting with relevant audio and video based instruction. The use of audio and video instruction within the study demonstrated promising results. Most notably the addition of the video component over previous non-video iterations showed improved results.

\section{The PIA Approach}

In contrast to the existing approaches used in SHs, the PIA project offers a relatively simple paradigm to provide assistance with ADL/IADLs. In PIA, the need for sensor deployment to detect activities is removed by instead relying on the use of relatively inexpensive NFC tags. Similarly the complexity associated with using an activity recognition process is removed by instead requiring an inhabitant to manually request assistance by activating the NFC tag with a smart device at the time and place they require it.

A number of works have used NFC within the domain of healthcare [16], [17], [18], [19], however, none have dealt directly with providing assistance with ADLs/IADLs.

\section{THE PIA SOLUTION}

Unlike approaches used by current prompting mechanisms, the use of easily recognizable NFC tags and smart devices allows the PIA solution to offer an easy to use and on demand approach to obtain assistance.

PIA is intended to provide support in a residential environment. With this approach there are two main categories of users; caregivers and users. Caregivers are typically friends, family or contracted assistance, however, may be any other trusted party.

Caregivers using the PIA system affix NFC tags to objects in a home (e.g. a washing machine). Then, using a smart device that has the PIA app installed, they record a video illustrating how to complete an activity associated with a specific NFC tag. After recording this instructional video the caregiver uploads the video clip to the PIA cloud and links the tag to the video using via a reasoning system. The PIA app provides a simple interface and workflow for caregivers. This interface is shown in Figure 1.

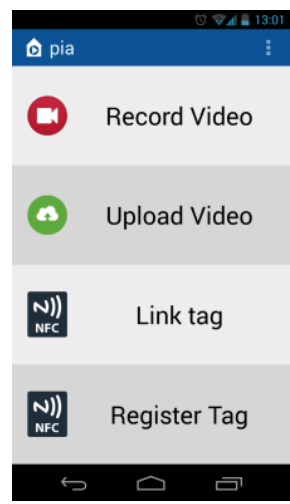

(a)

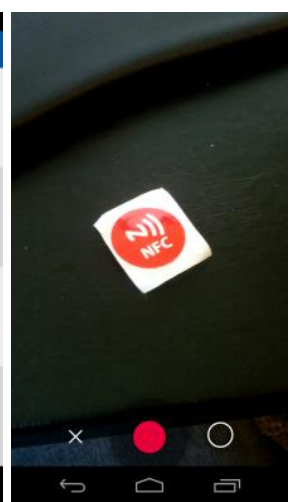

(b)

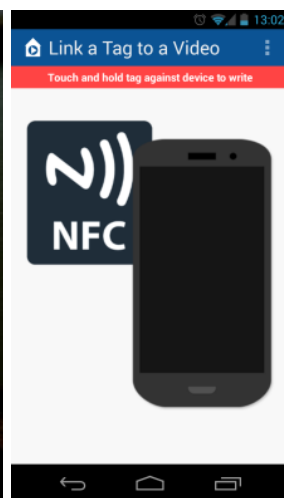

(c)
Figure 1. Screen shots of the menu (a), video recording (b) and tag linking (c) interface of the implemented PIA app.

Users in the PIA system are equipped with a smart device which has an instance of the same PIA app installed. If a user requires assistance they simply touch the smart device to an associated NFC tag, providing a simple avenue to gain assistance. The smart device interacts with the deployed NFC tag and reads its unique PIA ID. The ID is sent to the reasoning system to retrieve the URI of an associated video to download it from the cloud storage service and cache it on the device in order to automatically play it.

The combination of NFC tags, smart devices and a common app reflects the aim of PIA to provide a relatively 
inexpensive and low complexity method of providing assistance.

PIA uses a number of cloud-based components. These components provide increased flexibility and functionality of the PIA system. The main components include the reasoning system and the video repository.

The implemented architecture of the PIA Project, as depicted in Figure 2, allows these cloud components to be changed and expanded upon as required.

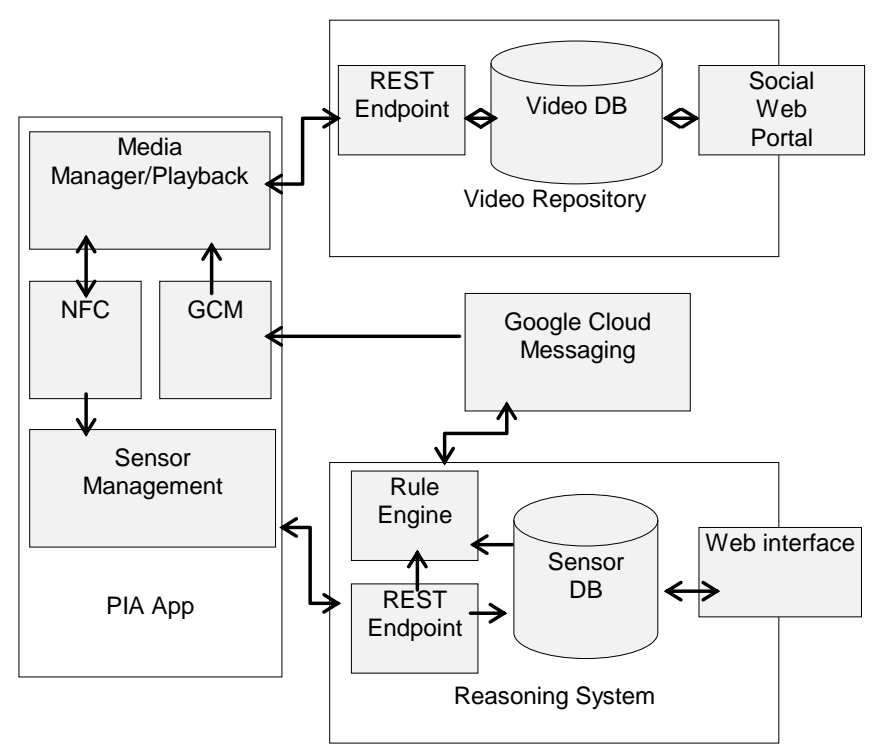

Figure 2. The architecture of the PIA project.

The reasoning component provides a rule based system for associating tags with videos. This reasoning system uses unique PIA IDs which are stored on deployed NFC tags in an environment and registered to its database.

When linking a tag using the app a default association rule is used to link the tag to a specific video allowing a low friction experience for the caregiver by not needing to explicitly create a rule. This allows a caregiver to assistance using only the PIA app.

On user interaction with an NFC tag its PIA ID is retrieved and sent to the reasoning service along with a Google Cloud Messaging (GCM) ID using web service communication for that particular device.

On receipt of the PIA ID and GCM ID the rule based reasoning system is used to determine the appropriate response to send to the smart device which provided the GCM ID. This response is sent to the device also using the GCM system. This response typically contains the URI of a video from the video repository.

Incorporating the reasoning system within the overall architecture provides a flexible mechanism of providing assistance based on rules allowing programmatic variation of a response for tag interactions. This flexibility can allow tailoring the instruction provided to a user depending on a number of factors such as the time of day or previous interaction with other tags in a residence.

In a scenario involving an NFC tag affixed to a coffee maker, for example, URIs of video instruction relevant to the time of day when the tag was activated can be delivered. For example, in the morning a user may prefer an espresso coffee and in the evening a milk-based coffee may be preferable.

In addition, rules can be defined to provide alerts to specified persons when necessary. This can provide the facility for an alert to be sent to a caregiver if an anomalous behavior rule is encountered. For example, such a rule could be triggered if a user required repeated assistance with a medication task within a certain time frame, which could indicate difficulty administering medication or the possibility of an overdose occurring.

These rules can be modified and created using the web interface of the reasoning system.

The video repository provides cloud based video storage, media processing, a Content Delivery Network (CDN) and a social web portal. This component produces multiple versions of videos for the PIA system in order to provide formats that are suitable for a multitude of devices, screen sizes and connection bandwidths in order to provide a better user experience. The CDN allows scalable distribution of video, subsequently removing a potential bottleneck PIA cloud.

The social web portal provides the social network features of the PIA system. This social network provides caregivers membership of communities. Caregivers in a community may collaborate, share and comment on instructional videos. This allows caregivers to improve the quality of instruction and care that they provide to users.

\section{INITIAL FINDINGS}

The PIA concept, along with a functional prototype of the PIA application deployed on a number of smart devices, was presented to focus groups. These focus groups were located in Germany, Norway and the UK and consisted of professional and family caregivers and older people (in the capacity of a proxy target user group). Some of the older people had memory problems and a small number had been diagnosed with dementia. A total of 41 individuals (11 caregivers, 30 users) contributed to the findings of these focus groups.

These focus groups used presentations in conjunction with a variety of smart devices running the PIA app to demonstrate the concept in order to determine user and caregiver acceptance. Among the findings some of the most pertinent were identified as:

-the focus groups found that the older persons and caregivers had a clear understanding of the overall aim of PIA and felt that it could be beneficial for persons suffering from memory problems, challenged by how to use new items around the home, or having difficulty maintaining their independence.

-the majority of caregivers believed that the PIA approach would be beneficial, however, not for those with advanced dementia. Instead, it was thought that it would be more useful for those with memory loss problems and early stage dementia. This belief is based on caregiver experience caring for individuals suffering from these types of problems. Specifically, difficulty can occur when technologies used to assist users are not familiar to, or compatible with, experiences that the user has had before onset of the underlying memory problems. 
Specific concerns raised were related to the ability of the target users to recognize the unfamiliar NFC tags in their environment and then use these tags in tandem with a smart device in order to obtain assistance. These concerns may be addressed by printing custom, highly recognizable and PIA themed, NFC tags and by using a bracket and caddy combination to ease device alignment.

An additional concern involved the potential distracting nature of using such a platform. It was reported that users could be distracted by using the systems and following a video to the extent that they may be unable to concentrate on the task.

Additionally, concerns were raised about the suitability of such an approach for those suffering from diminished dexterity due to afflictions such as arthritis.

Finally, the focus groups listed use scenarios beyond the initially presented functionality highlighting the flexibility of this this approach. These included the use of the PIA solution as a potential medication reminder platform and providing a mechanism for maintaining diet and grocery shopping.

\section{CONCLUSIONS AND Future WORK}

Using this approach the PIA project aims to offer an avenue of providing assistance with the daily tasks of a user thus providing greater independence. Furthermore, this assistance is offered in a manner which offers video based guidance from a familiar or trusted individual.

Unlike other solutions, which are reliant on complex sensor deployments within an environment, PIA uses NFC tags. NFC tags are relatively inexpensive and are easy to affix to items in a residence. This allows a relatively simple infrastructure for providing assistance with ADLs/IADLs.

In addition, this approach does not require any complex activity recognition mechanism to function. This provides a support system that is suitable for useful deployment in its current form, however, with a disadvantage as the users need to be aware of how to use the NFC tags and smart devices.

Focus groups evaluated the PIA approach and have commented that is shows promise assisting with IADLS in certain use cases where, generally, the user has the ability to use this technological solution.

Future versions of the PIA project will incorporate an increased amount of web based tools, including a refined version of the social network, and analysis tools. These analysis tools should cover IADL analysis and caregiver stress analysis to be used as a quality of life metric for both caregivers and users. Finally, feedback from focus groups will be incorporated into future iterations of the PIA project.

\section{ACKNOWLEDGMENT}

This work has been conducted in the context of the EU AAL PIA project (AAL-2012-5-033). The authors gratefully acknowledge the contributions from all members of the PIA consortium.

\section{REFERENCES}

[1] United Nation, World Population Ageing 2009 (Population Studies Series), Pap/Cdr Ed. 2010.
[2] d'Alessandro E. De Luca, S. Bonacci, and G. Giraldi, "Aging populations: the health and quality of life of the elderly.," $L a$ Clinica Terapeutica, vol. 162, no. 1, p. e13, 2011.

[3] D. J. Cook and S. K. Das, "How smart are our environments? An updated look at the state of the art," Pervasive and Mobile Computing, vol. 3, no. 2, pp. 53-73, Mar. 2007.

[4] M. Chan, D. Estève, C. Escriba, and E. Campo, "A review of smart homes- present state and future challenges.," Computer methods and programs in biomedicine, vol. 91, no. 1, pp. 55-81, Jul. 2008.

[5] G. Acampora, D. J. Cook, P. Rashidi, and A. V Vasilakos, "A Survey on Ambient Intelligence in Health Care.," Proceedings of the IEEE. Institute of Electrical and Electronics Engineers, vol. 101, no. 12, pp. 2470-2494, Dec. 2013.

[6] M. P. Poland, C. D. Nugent, H. Wang, and L. Chen, "Smart Home Research:Projects and Issues," International Journal of Ambient Computing and Intelligence, vol. 1, no. 4, pp. 32-45, Jan. 2009.

[7] S. Katz, A. Ford, and R. Moskowitz, "Studies of illness in the aged: the index of ADL: a standardized measure of biological and psychosocial function," Jama, vol. 185, no. 12, pp. 914-919, 1963.

[8] M. Lawton and E. Brody, "Instrumental Activities of Daily Living Scale (IADL)," 1988.

[9] L. Chen, J. Hoey, C. D. Nugent, D. J. Cook, and Z. Yu, "SensorBased Activity Recognition," IEEE Transactions on Systems, Man, and Cybernetics, Part C (Applications and Reviews), pp. $1-19,2012$.

[10] J. Hoey, P. Poupart, A. Von Bertoldi, T. Craig, C. Boutilier, and A. Mihailidis, "Automated handwashing assistance for persons with dementia using video and a partially observable Markov decision process," Computer Vision and Image Understanding, vol. 114, no. 5, pp. 503-519, May 2010.

[11] V. Perilli, G. E. Lancioni, F. Hoogeveen, A. Caffó, N. Singh, M. O’Reilly, J. Sigafoos, G. Cassano, and D. Oliva, "Video prompting versus other instruction strategies for persons with Alzheimer's disease.," American journal of Alzheimer's disease and other dementias, vol. 28, no. 4, pp. 393-402, Jun. 2013.

[12] A. Hwang and J. Hoey, "Smart home, the next generation: Closing the gap between users and technology," AAAI Fall Symposium on Gerontechnology. Arlington, VA, pp. 14-21, 2012.

[13] J. Lapointe, B. Bouchard, and J. Bouchard, "Smart homes for people with Alzheimer's disease: adapting prompting strategies to the patient's cognitive profile," Proceedings of the 5th International Conference on PErvasive Technologies Related to Assistive Environments, vol. 3, 2012.

[14] S. a. O'Neill, S. Mason, G. Parente, M. P. Donnelly, C. D. Nugent, S. McClean, B. Scotney, and D. Craig, "Video Reminders as Cognitive Prosthetics for People with Dementia," Ageing International, vol. 36, no. 2, pp. 267-282, Nov. 2010.

[15] K. Zingmark, "Promoting a good life among people with Alzheimer's disease," Journal of Advanced Nursing, vol. 38, no. 1, pp. 50-58, Apr. 2002.

[16] a. J. Jara, P. Lopez, D. Fernandez, M. a. Zamora, B. Ubeda, and a. F. Skarmeta, "Communication Protocol for Enabling Continuous Monitoring of Elderly People through Near Field Communications," Interacting with Computers, vol. 26, no. 2, pp. 145-168, May 2013.

[17] M. Vergara, P. Díaz-Hellín, J. Fontecha, R. Hervás, C. SánchezBarba, C. Fuentes, and J. Bravo, "Mobile Prescription: An NFCBased Proposal for AAL," 2010 Second International Workshop on Near Field Communication, pp. 27-32, 2010.

[18] J. Bravo, D. López-De-Ipiña, and C. Fuentes, "Enabling NFC technology for supporting chronic diseases: A proposal for alzheimer caregivers," Ambient Intelligence, pp. 109-125, 2008. D. López-de-Ipiña, I. Díaz-de-Sarralde, and J. Zubía, “An Ambient Assisted Living Platform Integrating RFID Data-on-Tag Care Annotations and Twitter.," Journal of Universal Computer Science, vol. 16, no. 12, pp. 1521-1538, 2010. 